\title{
Kawasaki Disease, Multisystem Inflammatory Syndrome in Children: Antibody-Induced Mast Cell Activation Hypothesis
}

\author{
Darrell O. Ricke ${ }^{1 *}$, Nicole Gherlone ${ }^{2}$, Philip Fremont-Smith', Philip Tisdall ${ }^{3}$, Maurice Fremont-Smith ${ }^{2}$ \\ 'MIT Lincoln Laboratory, USA \\ ${ }^{2}$ Frank H. Netter MD School of Medicine - Quinnipiac University, USA \\ ${ }^{3}$ Medical School Companion LLC, USA
}

Article Info

\section{Article Notes}

Received: June 02, 2020

Accepted: June 18, 2020

\section{*Correspondence:}

Dr. Darrell O. Ricke, MIT Lincoln Laboratory, USA; Telephone No: 1-781-999-5615; Email: Darrell.Ricke@ll.mit.edu.

C 2020 Ricke DO. This article is distributed under the terms of the Creative Commons Attribution 4.0 International License.

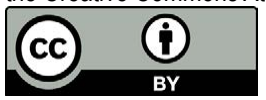

\section{Keywords:}

Multisystem Inflammatory Syndrome in Children

MIS-C

Kawasaki Disease

Mast Cells

Histamine

Mast Cell Activation Syndrome

MCAS

Aneurysm

\section{Abstract}

Multisystem Inflammatory Syndrome in Children (MIS-C) is appearing in infants, children, and young adults in association with COVID-19 (coronavirus disease 2019) infections of SARS-CoV-2. Kawasaki Disease (KD) is one of the most common vasculitides of childhood. KD presents with similar symptoms to MIS-C especially in severe forms such as Kawasaki Disease Shock Syndrome (KDSS). The observed symptoms for MIS-C and KD are consistent with Mast Cell Activation Syndrome (MCAS) characterized by inflammatory molecules released from activated mast cells. Based on the associations of KD with multiple viral and bacterial pathogens, we put forward the hypothesis that $\mathrm{KD}$ and MIS-C result from antibody activation of mast cells by Fc receptorbound pathogen antibodies causing a hyperinflammatory response upon second pathogen exposure. Within this hypothesis, MIS-C may be atypical KD or a KD-like disease associated with SARS-CoV-2. We extend the mast cell hypothesis that increased histamine levels are inducing contraction of effector cells with impeded blood flow through cardiac capillaries. In some patients, pressure from impeded blood flow, within cardiac capillaries, may result in increased coronary artery blood pressure leading to aneurysms, a well-known complication in KD.

Multisystem Inflammatory Syndrome in Children (MIS-C, previously designated as Pediatric Multisystem Inflammatory Syndrome - PMIS) is appearing in infants, children, and young adults in association with COVID-19 (coronavirus disease 2019) infections ${ }^{1-11}$. Kawasaki Disease (KD, previously called mucocutaneous lymph node syndrome) is one of the most common vasculitides of childhood ${ }^{12}$. KD presents with similar symptoms to MIS-C especially in severe forms such as Kawasaki Disease Shock Syndrome (KDSS). The cause of KD is currently unknown; KD has features similar to those associated with a viral infection. The leading hypothesis is that a ubiquitous infectious agent can induce KD in a genetically susceptible patient ${ }^{13}$. This hypothesis is supported by the presence of IgA plasma cells identified in inflamed tissues and coronary arteries of KD patients ${ }^{14}$. Associations between KD and multiple pathogens have been reported, including adenovirus ${ }^{15,16}$, human bocavirus ${ }^{17}$, coronavirus $^{16}$, human coronavirus $229 \mathrm{E}^{18}$, human coronavirus (HCoV-NH) NL63 ${ }^{19}$, cytomegalovirus ${ }^{20}$, dengue $^{21,22}$, enterovirus ${ }^{16,23}$, Epstein-Barr virus ${ }^{24}$, human herpesvirus $6^{25}$, human lymphotropic virus ${ }^{26}$, human rhinovirus ${ }^{16}$, influenza ${ }^{27}$, measles $^{28}$, parvovirus B19 ${ }^{29,30}$, parainfluenza virus type $2^{31}$, respiratory syncytial virus $(\mathrm{RSV})^{32}$, rotavirus ${ }^{33}$, varicella zoster

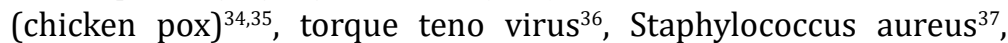
and Streptococcus ${ }^{24,38}$. Postinfluenza vaccination KD has also been 
reported $^{39}$. The seasonality and temporal clustering of $\mathrm{KD}^{32,40}$ further support an infectious etiology. A mild cold may precede the onset of KD and up to one-third of patients have concurrent, confirmed infections at the time of KD diagnosis ${ }^{41}$. The aggregate of these pathogen associations with KD supports the rejection of the hypothesis that KD is caused by a single infectious agent. The alternative hypothesis is that KD is associated with multiple infectious agents. We hypothesize that MIS-C may be atypical KD or a KD-like disease associated with SARS-CoV- $2^{42}$ as a result of antibody-dependent enhancement activation of mast cells. We further hypothesize that KD and MIS-C may be induced in part by histamine and other inflammatory molecules released from activation of mast cells by Fc receptor-bound pathogen antibodies resulting in a hyperinflammatory response.

The diagnosis of classic KD is based on the presence of fever lasting five days or longer together with at least four of five additional clinical findings: bilateral conjunctivitis, oral mucosal changes, cervical lymphadenopathy, extremity changes, and a polymorphous rash. However, not all patients present with this complete clinical picture. Atypical KD occurs in patients with fever lasting five days or longer with two or three of the previously mentioned clinical features. MIS-C symptoms demonstrate remarkable overlap (Table 1) and patients can meet criteria for atypical

Table 1. Similarities between MIS-C, KD, and KDSS (a subtype of KD)

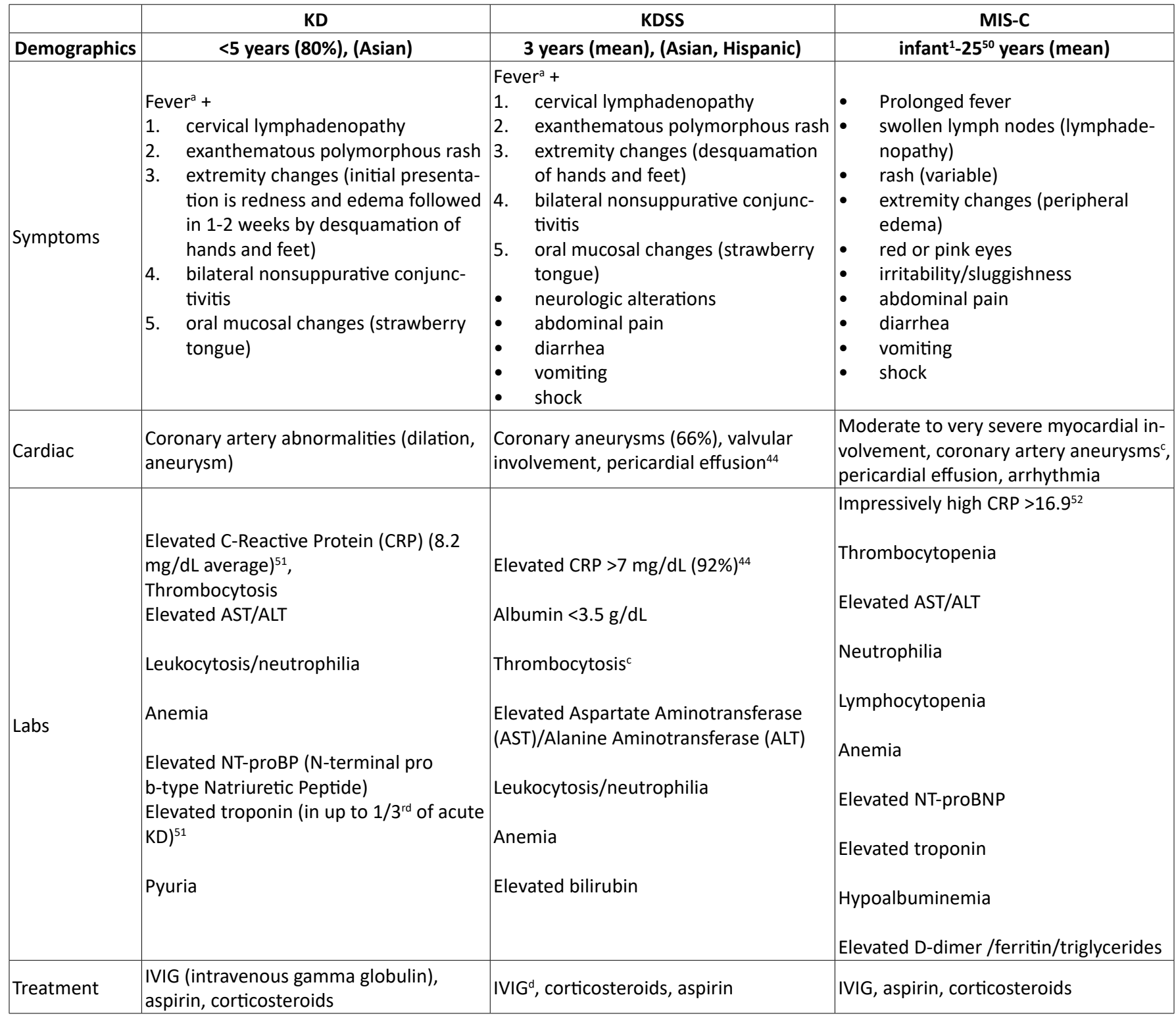

a5 consecutive days without identifiable source

${ }^{\mathrm{b}} \mathrm{MIS}-\mathrm{C}$ patients with coronary abnormalities may have had KD and were misclassified

cin one study, $54 \%$ of patients presented with thrombocytopenia

${ }^{\mathrm{d}}$ Higher incidence of IVIG resistance 
$\mathrm{KD}^{43}$. Accurate diagnosis is also complicated by difficulties distinguishing early KD symptoms from any common skin rash. As KD progresses, complications such as coronary artery aneurysms, heart failure, myocardial infarction, arrhythmias, and peripheral arterial occlusions may develop and lead to significant morbidity and mortality. KDSS is a complication of KD resulting in shock and hypotension. KDSS can present with multi-organ dysfunction and is associated with more severe inflammatory markers and coronary artery abnormalities ${ }^{44}$ similar to the clinical presentation of MIS-C. The observed symptoms for MIS-C and KD are consistent with Mast Cell Activation Syndrome (MCAS) characterized by inflammatory molecules released from activated mast cells. Pathogen binding to antibodies already attached to Fc receptors can activate mast cells, leading to the release of histamine and other compounds. Elevated histamine levels can lead to smooth muscle cell contraction in various organs, vasodilation, increased vascular permeability, and increased gastric acid section. This would result in clinical findings such as tachycardia, hypotension, erythema, edema, arrhythmias, urticaria, and diarrhea. Histamine also causes contractions of endothelial and pericyte cells ${ }^{45}$ resulting in impeded blood flow through capillaries; this is well characterized for cerebral blow flow following ischemic events ${ }^{46}$; we illustrate with available COVID Digital Pathology Repository ${ }^{47}$ examples from putative COVID-19 patients (Figure 1). We hypothesize that both COVID-19 patients and MIS-C patients may have the same impeded blood flow through capillaries likely due to increased histamine levels. Impeded blood flow in capillaries is likely the cause of myocarditis, valvulitis, necrotizing microvasculitis, edema of hands and feet, etc. In some patients, pressure from impeded blood flow within cardiac capillaries may result in increased coronary artery blood pressure leading to aneurysms, a well-known complication in KD (Figure 1). We hypothesize that KD and MIS-C coronary artery aneurysms are initiated with a pathologic dilation injury caused by increased pressure in the artery; the injured cells use damage-associated molecular patterns (DAMPs) signaling that initiates the inflammation at the site of injury. In this model, inflammation is a secondary event not the primary event leading to aneurysms. This inflammation is a feedforward process contributing to further weakening of the arterial wall. Of all arteries, the coronary artery has the shortest distance to downstream capillaries. Hypertension has been implicated in the pathogenesis of arterial aneurysms ${ }^{48,49}$.

Pediatric patients with first COVID-19 infection generally have less severe clinical manifestations than adult patients ${ }^{53}$. The COVID-19 asymptomatic infection rate has been estimated in adults to be $46 \%{ }^{54}$ and $46 \%$ in children in Hubei, China ${ }^{55}$. The COVID-19 outbreak in New York started mid-March, 2020. Children with MIS-C symptoms similar to KD associated with COVID-19
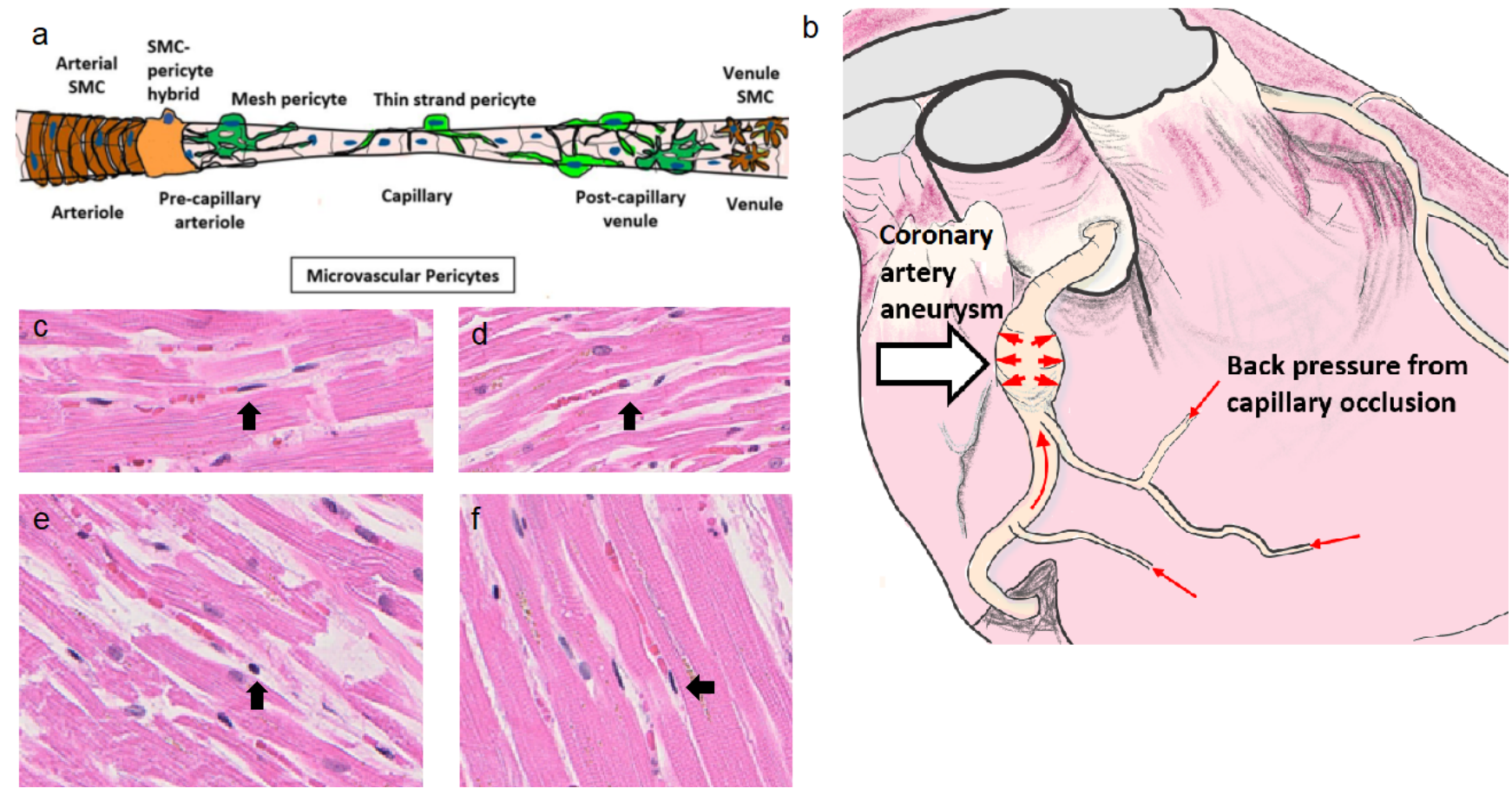

Figure 1: Back pressure model for coronary artery aneurysms from intra-myocardial capillary pericyte clamping post histamine stimulation. (a) Model of microvascular region for pericyte cell occlusion (b) coronary artery aneurysm caused by increased pressure from capillaries with impeded blood flow, (c-f) COVID Digital Pathology Repository putative COVID-19 ${ }^{47}$ patients' heart H\&E tissues with contracted effector cells (black arrows) (c) patient 9, (d) patient 6, (e) patient 8, and (f) patient 7. 
infections have been reported since late April, $2020^{56}$ suggesting that MIS-C manifests more than one month after the peak of COVID-19 cases in an affected area ${ }^{57}$. The number of affected children is rising rapidly; within weeks of the publication of COVID-19 related MIS-C cases ${ }^{2}, 93$ children were diagnosed with up to five deaths in New York. As of May $29^{\text {th }}, 186$ cases have been identified ${ }^{58}$. With an observed New York population infection rate of roughly $1.7 \%\left(337,055\right.$ infected $^{59}$ of $19,450,000$ population), the timing is consistent with a possible second wave of pediatric SARS-CoV-2 infections (1.7\% x $1.7 \%$ of 1.7 million New York children, or 491 possible second infections in New York children). With a rate of 306 cases per 100,000 under age 18 adjusted to 567 for asymptomatic children, the $1.7 \%$ infection rate of 1.7 million New York children predicts 169 possible second infections. The 186 cases identified by May $29^{\text {th }}$ represents between $110 \%$ of the lower estimate of 169 infections to $38 \%$ of the 491 that would be predicted in the second wave of infections. This second wave hypothesis is consistent with the lack of MIS-C patients in China and elsewhere that contained outbreaks in their regions consequently avoiding or minimizing the second wave of infections. With more than 250 cases of MIS-C reported in the United States by May 21, 202060, MIS-C may reflect a subset of children initially infected with SARS-CoV-2 who sustained a subsequent exposure to the virus. MIS-C may be a manifestation of a delayed trigger from the antibody-dependent disease. Without an effective vaccine for COVID-19, the first exposure to COVID-19 may result in asymptomatic to mild disease for most children accompanied by risk for MIS-C/KD upon subsequent infection. Ricke and Malone ${ }^{61}$ predict antibody-dependent enhancement disease risks associated with COVID-19 based on disease enhancements from animal model vaccine studies of severe acute respiratory syndrome, Middle East respiratory syndrome, and other coronaviruses. Extending this hypothesis to these children predicts that this postinfectious inflammatory process (MIS-C or KD) could be a direct result of antibody-dependent enhancement (ADE) of disease for children previously infected with SARS-CoV-2 or primary infected infants with either transplacental transferred antibodies (matAbs) or SARS-CoV-2 antibodies from breast milk. Children diagnosed with a postinfectious inflammatory process (MIS-C or KD) frequently test negative on PCR for SARS-CoV-2 ${ }^{1}$, however, they test positive for antibodies of SARS-CoV-2 ${ }^{52,57}$ suggesting antibodies are required for disease manifestation.

The symptoms of MIS-C and KD are consistent with the activation of mast cells via antibodies as seen in Mast Cell Activation Syndromes (MCAS). We hypothesize that SARS2 antibodies created after first infection bind mast cells, triggering histamine release. These antibodies may be recognizing low levels of SARS-CoV-2 from a re-emergent infection or a possible second exposure. Mast cell released histamine stimulates pericytes or effector cells causing capillary constriction, notably in cardiac tissue. The MIS-C/ KD symptoms of diarrhea and vomiting may be attributed to increased histamine levels and/or SARS-CoV-2 gastrointestinal infection akin to symptoms seen for the severe acute respiratory syndrome. Current KD and MIS-C treatments include intravenous gamma globulin (IVIG), corticosteroids, and high-dose aspirin ${ }^{62}$. IVIG can decrease, but not eliminate, this risk of developing coronary artery aneurysms. Corticosteroids as adjunctive therapy to IVIG may be helpful for lowering the risk of coronary artery aneurysms ${ }^{62}$. We hypothesize that IVIG dilutes the current infection pathogen antibodies bound to mast cells, reducing the level of mast cell activation. High doses of aspirin may help treat inflammation; aspirin can also decrease pain and reduce fever. While not appreciated for treating $\mathrm{KD}$, aspirin is a known mast cell stabilizer. This ADE model for MIS-C and COVID-19 emphasizes the importance of developing safe T-cell vaccines and increases the importance of safety testing for any B-cell vaccines being developed. In addition to IVIG, famotidine ${ }^{63}$, famotidine +cetirizine ${ }^{64}$, montelukast ${ }^{65}$ and other potential treatments have been identified by Malone et al. ${ }^{66}$ to reduce the degranulation of mast cells. We suggest children presenting with evidence of MIS-C/KD should also be tested for antibodies for SARS$\mathrm{CoV}-2$ as PCR testing is frequently negative.

\section{Conclusion}

We must be cautious of inappropriately creating new clinical entities in the context of COVID-1967. It has been hypothesized before the advent of COVID-19 that KD could be induced by RNA viruses in genetically susceptible hosts. It is also possible we are seeing SARS-CoV-2 induced KD complicated by KDSS in MIS-C as a result of antibodydependent enhancement activation of mast cells.

\section{Conflict of Interest}

The authors have indicated they have no potential conflicts of interest to disclose.

\section{Acknowledgements}

The authors acknowledge the Department of Defense(DoD), Defense Threat Reduction Agency(DTRA), and The Joint Science and Technology Office(JSTO) of the Chemical and Biological Defense Program (CBDP) for their support under the Discovery of Medical countermeasures Against Novel Entities (DOMANE) initiative. The authors acknowledge Nora Smith for literature search assistance. DR acknowledges Adam Michaleas for COVID-19 research advocacy. DR gratefully acknowledges the support of the MIT SuperCloud team.

DISTRIBUTION STATEMENT A. Approved for public release. Distribution is unlimited. 
This material is based upon work supported under Air Force Contract No. FA8702-15-D-0001. Any opinions, findings, conclusions or recommendations expressed in this material are those of the author(s) and do not necessarily reflect the views of the U.S. Air Force.

\section{References}

1. Jones VG, Mills M, Suarez D, et al. COVID-19 and Kawasaki Disease: Novel Virus and Novel Case. Hosp Pediatr hpeds. 2020; 2020-0123, doi:10.1542/hpeds.2020-0123.

2. Russo M. Up to 5 NY Children Dead, 93 Sickened by Rare COVIDRelated Illness. 2020. https://www.nbcnewyork.com/investigations/ kawasaki-disease-up-to-5-ny-children-dead-85-sickened-by-rarecovid-related-illness/2411571/. Accessed May 26, 2020

3. Belhadjer Z, Meot M, Bajolle F, et al. Acute heart failure in multisystem inflammatory syndrome in children (MIS-C) in the context of global SARS-CoV-2 pandemic. Circulation. 2020, doi:10.1161/ CIRCULATIONAHA.120.048360.

4. Cheung EW, Zachariah P, Gorelik M, et al. Multisystem Inflammatory Syndrome Related to COVID-19 in Previously Healthy Children and Adolescents in New York City. JAMA. 2020. doi:10.1001/ jama.2020.10374.

5. Rivera-Figueroa EI, Santos R, Simpson S, et al. Incomplete Kawasaki Disease in a Child With Covid-19. Indian Pediatr. 2020.

6. Chiotos K. Multisystem Inflammatory Syndrome in Children During the Coronavirus 2019 Pandemic: A Case Series. J Pediatric Infect Dis Soc. 2020. doi:10.1093/jpids/piaa069.

7. Deza Leon MP, Redzepi A, McGrath E, et al. COVID-19-Associated Pediatric Multisystem Inflammatory Syndrome. J Pediatric Infect Dis Soc. 2020. doi:10.1093/jpids/piaa061.

8. Rauf A, Vijayan A, John ST, et al. Multisystem Inflammatory Syndrome With Features of Atypical Kawasaki Disease During COVID-19 Pandemic. Indian J Pediatr. 2020.

9. $\mathrm{Xu} \mathrm{S}$, Chen M, Weng J. COVID-19 and Kawasaki disease in children Pharmacol Res. 2020; 159: 104951-104951, doi:10.1016/j. phrs.2020.104951.

10. Moreira A. Kawasaki disese linked to COVID-19 in children. Nat Rev Immuol. 2020.

11. Toubiana J, Poirault C, Corsia A, et al. Kawasaki-like multisystem inflammatory syndrome in children during the covid-19 pandemic in Paris, France: prospective observational study. BMJ 369. 2020; m2094 doi:10.1136/bmj.m2094.

12. Newburger JW, Takahashi M, Burns JC. Kawasaki Disease. J Am Coll Cardiol. 2016; 67: 1738-1749, doi:https://doi.org/10.1016/j. jacc.2015.12.073.

13. Shulman ST, Rowley AH. Kawasaki disease: insights into pathogenesis and approaches to treatment. Nat Rev Rheumatol. 2015; 11: 475-482, doi:10.1038/nrrheum.2015.54.

14. Soni PR, Noval Rivas M, Arditi MA Comprehensive Update on Kawasaki Disease Vasculitis and Myocarditis. Curr Rheumatol Rep. 2020; 22: 6, doi:10.1007/s11926-020-0882-1.

15. Embil JA, McFarlane ES, Murphy DM, et al. Adenovirus type 2 isolated from a patient with fatal Kawasaki disease. Can Med Assoc J. 1985; 132: $1400-1400$

16. Chang LY, Lu CY, Shao PL, et al. Viral infections associated with Kawasaki disease. J Formos Med Assoc. 2014; 113: 148-154, doi:10.1016/j.jfma.2013.12.008

17. Catalano-Pons C, Giraud C, Rozenberg F, et al. Detection of human bocavirus in children with Kawasaki disease. Clin Microbiol
Infect. 2007; 13: 1220-1222, doi:https://doi.org/10.1111/j.14690691.2007.01827.x

18. Shirato K, Imada Y, Kawase M, et al. Possible involvement of infection with human coronavirus 229E, but not NL63, in Kawasaki disease. Med Virol. 2014; 86: 2146-2153, doi:10.1002/jmv.23950.

19. Esper F, Weibel C, Ferguson D, et al. Evidence of a Novel Human Coronavirus That Is Associated with Respiratory Tract Disease in Infants and Young Children. J Infect Dis. 2005; 191: 492-498, doi:10.1086/428138.

20. Catalano-Pons C, Quartier P, Leruez-Ville $M$, et al. Primary Cytomegalovirus Infection, Atypical Kawasaki Disease, and Coronary Aneurysms in 2 Infants. Clin Infect Dis. 2005; 41: e53-e56, doi:10.1086/432578.

21. Jagadeesh A, Krishnamurthy S, Mahadevan S. Kawasaki Disease in a 2-year-old Child with Dengue Fever. Indian J Pediatr. 2016; 83: 602 603, doi:10.1007/s12098-015-1927-8.

22. Sopontammarak S, Promphan W, Roymanee S, et al. Positive Serology for Dengue Viral Infection in Pediatric Patients With Kawasaki Disease in Southern Thailand. Circ J. 2008; 72: 1492-1494, doi:10.1253/circj. CJ-08-0158.

23. Weng KP, Cheng-Chung Wei J, Hung YM, et al. Enterovirus Infection and Subsequent Risk of Kawasaki Disease: A Population-based Cohort Study. Pediatr Infect Dis J. 2018; 37.

24. Kikuta H, Nakanishi M, Ishikawa N, et al. Detection of EpsteinBarr virus sequences in patients with Kawasaki disease by means of the polymerase chain reaction. Intervirology. 1992; 33: 1-5, doi:10.1159/000150224.

25. Okano M, Luka J, Thiele GM, et al. Human herpesvirus 6 infection and Kawasaki disease. J Clin Microbiol. 1989; 27: 2379-2380.

26. Okano M. Kawasaki Disease and Human Lymphotropic Virus Infection. Curr Med Res Opin. 1999; 15: 129-134, doi:10.1185/03007999909113373.

27. Joshi AV, Jones KDJ, Buckley AM, et al. Kawasaki disease coincident with influenza A H1N1/09 infection. Pediatr Int. 2011; 53: e1-e2, doi:10.1111/j.1442-200X.2010.03280.x.

28. Whitby D, Hoad JG, Tizard EJ, et al. Isolation of measles virus from child with Kawasaki disease. Lancet. 1991; 338: 1215, doi:https:// doi.org/10.1016/0140-6736(91)92085-G.

29. Holm JM, Hansen LK, OxhojH. Kawasaki disease associated with parvovirus B19 infection. Eur J Pediatr. 1995; 154: 633-634, doi:10.1007/BF02079066

30. Nigro G, Zerbini M, Krzysztofiak A, et al. Active or recent parvovirus B19 infection in children with Kawasaki disease. Lancet. 1994; 343: 1260-1261, doi:https://doi.org/10.1016/S0140-6736(94)92154-7.

31. Keim D, Keller E, Hirsch M. Mucocutaneous Lymph-Node Syndrome and Parainfluenza 2 Virus Infection. Lancet. 1977; 310: 303, doi:https://doi.org/10.1016/S0140-6736(77)90990-4.

32. Kim GB, Park S, Kwon BS, et al. Evaluation of the Temporal Association between Kawasaki Disease and Viral Infections in South Korea Korean Circ J. 2014; 44: 250-254, doi:10.4070/kcj.2014.44.4.250.

33. Matsuno S, Utagawa E, Sugiura A. Association of Rotavirus Infection with Kawasaki Syndrome. I Infect Dis. 1983; 148: 177-177, doi:10.1093/infdis/148.1.177.

34. Ogboli MI, Parslew R, Verbov J, et al. Kawasaki disease associated with varicella: a rare association. Br J Dermatol. 1999; 141: 1136-1152, doi:10.1046/j.1365-2133.1999.03231.x

35. Kossiva L, Papadopoulos M, Lagona E, et al. Myocardial infarction in a 35-day-old infant with incomplete Kawasaki disease and chicken pox. Cardiol Young. 2010; 20: 567-570, doi:10.1017/ S1047951109991478. 
36. Thissen JB, Isshiki $\mathrm{M}$, Jaing $\mathrm{C}$, et al. A novel variant of torque teno virus 7 identified in patients with Kawasaki disease. PLoS One. 2018; 13 e0209683-e0209683, doi:10.1371/journal.pone.0209683.

37. Hall M, Hoyt L, Ferrieri P, et al. Kawasaki Syndrome-Like Illness Associated with Infection Caused by Enterotoxin B-Secreting Staphylococcus aureus. Clin Infect Dis. 1999; 29: 586-589, doi:10.1086/598638.

38. Shinomiya N, Takeda T, Kuratsuji T, et al. Variant Streptococcus sanguis as an etiological agent of Kawasaki disease. Prog Clin Biol Res. 1987; 250: 571-572.

39. Shimada S, Watanabe T, Sato SA Patient with Kawasaki Disease Following Influenza Vaccinations. Pediatr Infect Dis J. 2015; 34

40. Burns JC, Cayan DR, Tong G, et al. Seasonality and temporal clustering of Kawasaki syndrome. Epidemiol. 2005; 16: 220-225, doi:10.1097/01. ede.0000152901.06689.d4

41. Benseler SM, McCrindle BW, Silverman ED, et al. Infections and Kawasaki Disease: Implications for Coronary Artery Outcome. Pediatr 2005; 116: e760, doi:10.1542/peds.2005-0559.

42. Verdoni L, Mazza A, Gervasoni A, et al. An outbreak of severe Kawasaki-like disease at the Italian epicentre of the SARS-CoV-2 epidemic: an observational cohort study. Lancet. 2020, doi:10.1016/ S0140-6736(20)31103-X

43. Deza Leon MP, Redzepi A, McGrath E, et al. COVID-19 Associated Pediatric Multi-System Inflammatory Syndrome. J Pediatric Infect Dis Soc. 2020, doi:10.1093/jpids/piaa061.

44. Gamez-Gonzalez LB, Moribe-Quintero I, Cisneros-Castolo M, et al. Kawasaki disease shock syndrome: Unique and severe subtype of Kawasaki disease. Pediatr Int. 2018; 60: 781-790, doi:10.1111/ ped.13614

45. Kelley C, D'Amore P, Hechtman HB, et al. Vasoactive hormones and cAMP affect pericyte contraction and stress fibresin vitro. J Muscle Res Cell Motil. 1988; 9: 184-194, doi:10.1007/BF01773740.

46. Attwell D, Mishra A, Hall CN, et al. What is a pericyte? J Cereb Blood Flow Metab. 2016; 36: 451-455, doi:10.1177/0271678X15610340.

47. COVID Digital Pathology Repository. 2020. https://covid19pathology. nih.gov/. Accessed June 14, 2020.

48. Spittell JA. Hypertension and arterial aneurysm. Journal of the American College of Cardiology. 1983; 1: 533-540, doi:https://doi org/10.1016/S0735-1097(83)80085-0.

49. Aortic Aneurysm. 2019 https://www.cdc.gov/heartdisease/ aortic_aneurysm.htm?CDC_AA_refVal=https $\% 3 \mathrm{~A} \% 2 \mathrm{~F} \% 2 \mathrm{Fwww}$. cdc.gov\%2Fdhdsp\%2Fdata_statistics\%2Ffact_sheets\%2Ffs_aortic_ aneurysm.htm. Accessed June 14, 2020.

50. MIS-C: Young adults also affected by Kawasaki-like disease. 2020. https://www.washingtonpost.com/health/2020/05/21/misc-ckawasaki-coronavirus-young-adults/. Accessed May 26, 2020.

51. Sato YZ, Molkara DP, Daniels LB, et al. Cardiovascular biomarkers in acute Kawasaki disease. Int J Cardiol. 2013; 164: 58-63, doi:https:// doi.org/10.1016/j.ijcard.2011.06.065.

52. Riphagen S, Gomez X, Gonzalez-Martinez C, et al. Hyperinflammatory shock in children during COVID-19 pandemic. Lancet. 2020; 395 : 1607-1608, doi:10.1016/S0140-6736(20)31094-1.

53. Dong Y. Epidemiological Characteristics of 2143 Pediatric Patients With 2019 Coronavirus Disease in China. Pediatr. 2020; e20200702, doi:10.1542/peds.2020-0702.

54. He W, Yi GY, Zhu Y. Estimation of the basic reproduction number average incubation time, asymptomatic infection rate, and case fatality rate for COVID-19: Meta-analysis and sensitivity analysis. medRxiv. 2020, doi:10.1101/2020.04.28.20083758.

55. Dong Y, Mo X, Hu Y, et al. Epidemiology of COVID-19 Among Children in China. Pediatr. 2020; e20200702, doi:10.1542/peds.2020-0702.

56. Fact Sheet: Pediatric Multisystem Inflammatory Syndrome. 2020. https://www1.nyc.gov/assets/doh/downloads/pdf/imm/covid-19pmis.pdf. Accessed May 26, 2020.

57. Shulman ST. Pediatric COVID-associated Multi-system Inflammatory Syndrome (PMIS). J Pediatric Infect Dis Soc. 2020, doi:10.1093/jpids/ piaa062.

58. Childhood Inflammatory Disese Related to COVID-19. 2020. https:// coronavirus.health.ny.gov/childhood-inflammatory-disease-relatedcovid-19. Accessed May 26, 2020.

59. COVID-19 Dashboard by the Center for Systems Science and Engineering (CSSE) at Johns Hopkins University (JHU). 2020. https:// gisanddata.maps.arcgis.com/apps/opsdashboard/index.html\#/ bda7594740fd40299423467b48e9ecf6. Accessed May 11, 2020.

60. Czachor E. More Than 250 Cases of Coronavirus-Linked Inflammatory Disease MIS-C Have Been Reported in the U.S. 2020, https://www. newsweek.com/more-250-cases-coronavirus-linked-inflammatorydisease-mis-c-have-been-reported-us-1505812. Accessed May 26, 2020

61. Ricke DO, Malone RW. Medical Countermeasures Analysis of 2019nCoV and Vaccine Risks for Antibody-Dependent Enhancement (ADE) Preprints. 2020.

62. Saguil A, Fargo M, Grogan S. Diagnosis and Management of Kawasaki Disease. Am Fam Physician. 2015; 91: 365-371.

63. Freedberg DE, Conigliaro J, Wang TC, et al. Famotidine Use is Associated with Improved Clinical Outcomes in Hospitalized COVID-19 Patients: A Propensity Score Matched Retrospective Cohort Study. Gastroenterology. 2020, doi:10.1053/j.gastro.2020.05.053.

64. Jackson CA. New COVID-19 clinical trial will utilize combination of two historically safe drugs. 2020, https://www.wlox.com/2020/06/09/ new-covid-clinical-trial-will-utilize-combination-two-historicallysafe-drugs/. Accessed June 14, 2020.

65. Fidan C, Aydogdu A. As a potential treatment of COVID-19: Montelukast. Med Hypotheses. 2020; 142: 109828-109828, doi:10.1016/j.mehy.2020.109828.

66. Malone RW. COVID-19: Famotidine, Histamine, Mast Cells, and Mechanisms. Preprint (Version1) at ResearchSquare. 2020, doi:10.21203/rs.3.rs-30934/v1.

67. Loomba RS, Villarreal E, Flores S. Covid-19 and Kawasaki syndrome: should we really be surprised? Cardiol Young. 2020; 1-5, doi:10.1017/ S1047951120001432. 
